PO040

\title{
IMPACT OF BACKGROUND FIELD SIZE AND CORNEAL ILLUMINANCE ON THE DEGREE OF CHROMATIC ADAPTATION
}

\author{
Shining Ma et al.
}

DOI 10.25039/x46.2019.PO040

from

CIE x046:2019

Proceedings

of the

29th CIE SESSION

Washington D.C., USA, June 14 - 22, 2019

(DOI 10.25039/x46.2019)

The paper has been presented at the 29th CIE Session, Washington D.C., USA, June 14-22, 2019. It has not been peer-reviewed by CIE.

(c) CIE 2019

All rights reserved. Unless otherwise specified, no part of this publication may be reproduced or utilized in any form or by any means, electronic or mechanical, including photocopying and microfilm, without permission in writing from CIE Central Bureau at the address below. Any mention of organizations or products does not imply endorsement by the CIE.

This paper is made available open access for individual use. However, in all other cases all rights are reserved unless explicit permission is sought from and given by the CIE.

CIE Central Bureau

Babenbergerstrasse 9

A-1010 Vienna

Austria

Tel.: +4317143187

e-mail: ciecb@cie.co.at

www.cie.co.at 


\title{
IMPACT OF BACKGROUND FIELD SIZE AND CORNEAL ILLUMINANCE ON THE DEGREE OF CHROMATIC ADAPTATION
}

\author{
Shining Ma ${ }^{1}$, Peter Hanselaer ${ }^{1}$, Kees Teunissen ${ }^{2}$, Kevin A.G. Smet ${ }^{1}$ \\ ${ }^{1}$ ESAT/Light\&Lighting Laboratory, KU Leuven, Ghent, BELGIUM, \\ ${ }^{2}$ Signify, High Tech Campus 7, Eindhoven, NETHERLANDS
}

shining.ma@kuleuven.be

DOI 10.25039/x46.2019.PO040

\begin{abstract}
Some chromatic adaptation transforms (CAT) include a parameter $(D)$ to indicate the degree of adaptation to the illumination (between 0 and 1 ). However, the $D$ formula in the most widely used CAT model - CAT02 - fails to predict the degree of adaptation in a complex scene because it is only a function of the background luminance and surround conditions. In this study, an achromatic matching experiment was conducted under 13 background chromaticities for 3 sizes of the adapting field $\left(20^{\circ}, 40^{\circ}, 60^{\circ}\right)$ under 3 different luminance levels resulting in the same corneal illuminance. Corresponding colour sets were derived from achromatic settings of a grey cube. The result showed that the CAT02 model indeed performs poorly for all background conditions and that a larger field of view generates a more complete adaptation, while the same luminous flux was received by the eyes and despite the very different adapting field luminance.
\end{abstract}

Keywords: CAT02, adapting field size, degree of adaptation, corneal illuminance, coloured illumination, achromatic matching, chromatic illumination

\section{Introduction}

Chromatic adaptation is an important visual mechanism for adapting to the changes in the colour (spectrum) of the illumination. As a result of chromatic adaptation (among others), objects tend to have nearly the same colour appearance in many illumination conditions, a phenomenon known as colour constancy. CIE recommended the CAT02 (CIE, 2004) chromatic adaptation transform (CAT) embedded in CIECAM02 (Moroney, 2002) to predict adaptive colour shifts. Although, not (yet) officially recommended by CIE, CAT02 and CIECAM02 were recently updated to CAT16 and CAM16 (Li, 2017;Li, 2018), respectively, to correct some mathematical problems (Brill, 2013). In this paper, CAT02 is still used, as the main focus is on the degree of adaptation $D$, not on the CAT sensor space.

The CAT02 model is a CAT of the von Kries (von Kries, 1902) type applied in a sharpened sensor space. It includes, an equation predicting the degree of adaptation $(D)$, which is, however, only related to the luminance of adapting field $\left(L_{A}\right)$, as shown below:

$$
D=F\left[1-\left(\frac{1}{3.6}\right) e^{\left(\frac{-L_{A}-42}{92}\right)}\right]
$$

where

$F \quad$ is a factor dependent on the surround conditions (1: 'average', 0,9: 'dim' and 0,8: 'dark')

$L_{A} \quad$ is the luminance $\left(\mathrm{cd} / \mathrm{m}^{2}\right)$ of the adapting field, typically considered the average luminance of the illuminated uniform background, surrounding the stimulus.

In a recent study the impact of field extent on the degree of adaptation was investigated for 3 field sizes with the same luminance level (Ma, 2018). The degree of adaptation was found to increase at larger field extents. However, the same background luminance, results in more light entering the eyes for a larger fields of view. Therefore, rather than the background field extent, it is possible that the luminous flux received at the eyes is the driving factor. This paper will report the results of a series of experiments with background fields of varying sizes, but with luminance values adjusted to result in equal vertical eye illuminance, henceforth referred to as corneal illuminance. 


\section{Method}

\subsection{Apparatus}

In the experiment, the test stimulus was a 3D grey (spectrally flat) cube centrally positioned in a white background scene, typically considered to be providing the adaptation field. The reflectance spectra of the background field and the grey cube are plotted in Figure 1, which was measured by a Hunterlab UltraScan Pro colorimeter. A calibrated data projector was used to provide independent and controllable illumination on the background and the stimulus. The reflected spectral radiance of the background and cube was measured by an OceanOptics QE65Pro tele-spectroradiometer. The size (visual angle) of the grey cube is $6^{\circ}$ and the field size (visual angle) of the background varied between $20^{\circ}$ and $60^{\circ}$. Therefore, the CIE $196410^{\circ}$ colour matching functions were adopted in all colorimetric calculations.

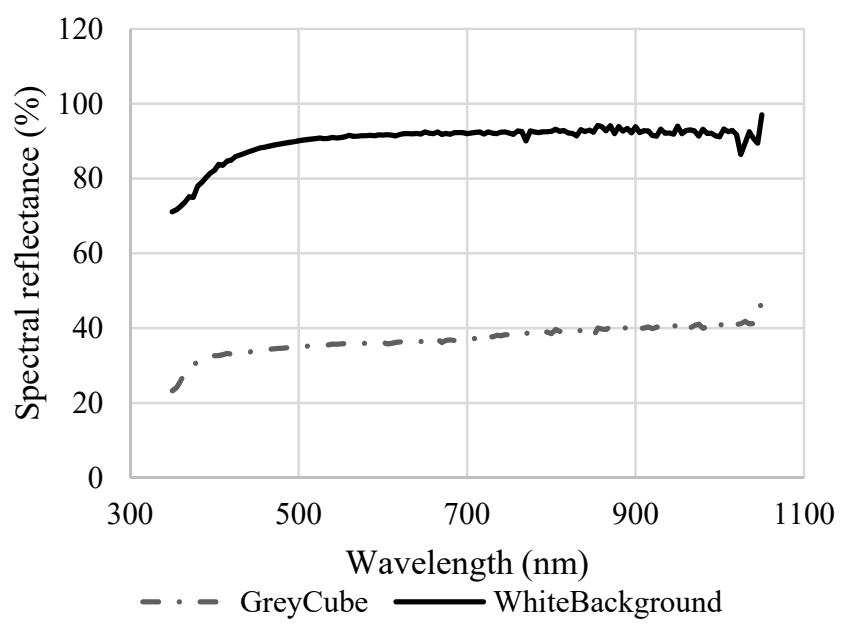

Figure 1 - The reflectance spectrum of the grey cube stimulus and white background adapting field

The experiment was conducted for 3 background field sizes: $20^{\circ}, 40^{\circ}, 60^{\circ}$ as shown in Figure 2. Each experiment session included one field size. The vertical illuminance measured at the eye position of observers was kept constant at $7 / x$ for all the adapting fields. The background luminance values for the three field extents were $180 \mathrm{~cd} / \mathrm{m}^{2}, 35 \mathrm{~cd} / \mathrm{m}^{2}$ and $20 \mathrm{~cd} / \mathrm{m}^{2}$, respectively. For each background size, the same thirteen background chromaticities were generated: three neutral ones (corresponding to EEW and D65, and the most neutral white $(\mathrm{N})$ found by Smet et al. (Smet, 2015)), five corresponding to Planckian radiators (illuminants $\mathrm{A}, 2000 \mathrm{~K}, 4000 \mathrm{~K}$, $12000 \mathrm{~K}$ and infinite $\mathrm{K}$ ), and five high chroma ones(Red, Yellow, Green, Blue, and magenta). These background adaptation chromaticities cover a large gamut in colour space.

(a)

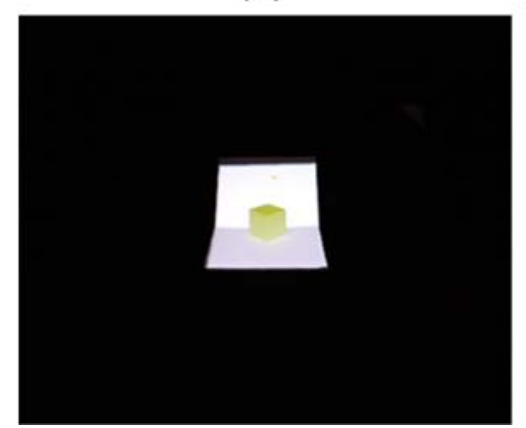

(b)

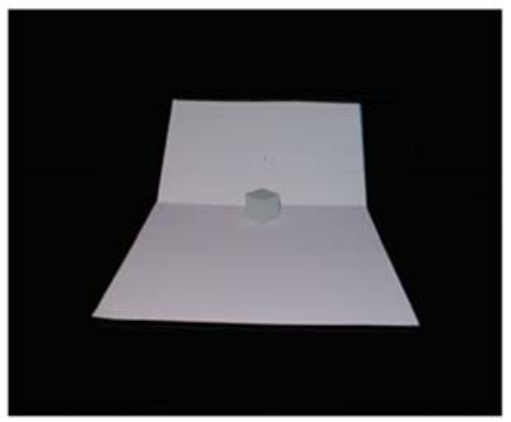

(c)

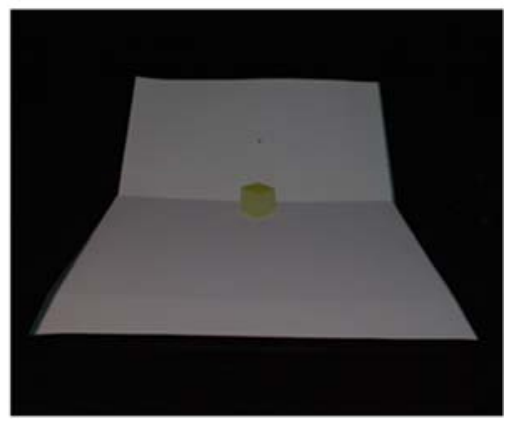

Figure 2 - Adapting field sizes and their luminance values. (a) $20^{\circ}$ with $180 \mathrm{~cd} / \mathrm{m}^{2}$ (b) $40^{\circ}$ with 35 $\mathrm{cd} / \mathrm{m}^{2}$ (c) $60^{\circ}$ with $20 \mathrm{~cd} / \mathrm{m}^{2}$. 


\subsection{Procedure}

An achromatic matching (AM) method has been used to collect corresponding colour sets for varying extents of the background (adaption) field illuminated by a number of neutral and coloured illuminations. Observers were required to adjust the colour appearance of the presented stimuli until it matched the target colour (neutral grey) in their memory.

During the experiments, the observers sat in front of the background field and centrally positioned neutral grey cube, with a distance of $2 \mathrm{~m}$ to the latter. They were asked to adjust the colour appearance of the cube to neutral grey by using the arrow keys on a regular keyboard. The four arrow keys (left/right and up/down,) correspond to stimulus adjustment along CIE 1976 $u^{\prime}$ and $v^{\prime}$ coordinate axes. Note that they needed to adapt for 45 seconds before starting their matching (Fairchild, 1995; Rinner, 2000). Then they needed to rate their satisfaction with the accuracy of their colour match on a 0 (not satisfied at all, i.e. couldn't make a good match) to 10 (very satisfied, i.e. could make a good match) scale. To minimize starting bias, each matching was repeated 4 times with 4 different initial chromaticities. The latter were evenly distributed along the hue circle centred at the chromaticity of the equal-energy white in CIE $1976 u^{\prime} 10 V^{\prime} 10$ chromaticity diagram. To avoid order bias, the background colours and stimulus starting points were presented randomly within a single experiment session and the order of three sessions was also randomized.

Eleven observers ( 7 females and 4 males, average age: $25 \pm 1,3$ years) with normal colour vision, as tested by the Ishihara 24 -plate test, participated in the experiments.

\section{Analysis}

\subsection{Observer variability}

Observer uncertainty and variability (intra- and inter-) were quantified by the MCDM (Mean Colour Difference from the Mean) (Billmeyer, 1981) in terms of colour difference in the CIE 1976 u' $^{\prime} 10 V^{\prime} 10$ chromaticity diagram. Note that intra-variability for each background chromaticity has been evaluated with the observer's average achromatic match, calculated from the 4 repeats, corresponding to the 4 starting points. The higher the $M C D M$, the larger the variation between observers (inter-variability), and the lower the repeatability or the consistency within observers (intra-variability). The mean inter- and intra-observer MCDM values calculated across all illumination conditions for $20^{\circ}, 40^{\circ}$ and $60^{\circ}$ adapting field are respectively 0,0097 , $0,0075,0,0094 u^{\prime}{ }_{10} v^{\prime}{ }_{10}$ units and 0,0095, 0,0105, 0,0081 $u^{\prime}{ }_{10} v^{\prime}{ }_{10}$ units.

\subsection{Model test of CAT02}

For each background field size, 12 sets of corresponding colour (CC) sets were derived from the achromatic matches made for each of the 13 background chromaticities by selecting the data for the EEW background chromaticity as the reference. The performance of the CAT02 model has been evaluated by calculating the CIE $1976 u^{\prime}{ }_{10} v^{\prime}{ }_{10}$ colour difference $\left(\Delta\left(u^{\prime}{ }_{10}, v^{\prime}{ }_{10}\right)\right)$ between the visual match and the one predicted by CAT02 using the CAT02 $D$-formula, which is only dependent on the luminance of the adapting field for a fixed value of the surround factor, $F(0,8$ for our experiment, i.e., dark surround). The calculated CAT02 $D$ values, for a dark surround condition, are equal to $0,78,0,70$ and 0,68 for the $20^{\circ}, 40^{\circ}$ and $60^{\circ}$ background field sizes, respectively. The CAT02 $D$ decreases with the increasing field of view as the luminance of adapting field decreases.

Figure 3 shows the CAT02 prediction error (colour difference) for the 13 background chromaticities under each of the three field sizes. Firstly, it can be seen that almost for all the background chromaticities, a larger field size corresponds to a lower prediction error. The $u^{\prime}{ }_{10} v^{\prime}{ }_{10}$ colour differences averaged over the $12 \mathrm{CC}$ sets are $0,0348,0,0232,0,0201$ for the $20^{\circ}$, $40^{\circ}$ and $60^{\circ}$ field sizes, respectively. This is approximately two to three times as large as the inter-observer variability, indicating substantial room for improvement in CAT predictive performance. Note that the prediction error decreases as the field size becomes larger, indicating that the degree of adaptation is better estimated under larger backgrounds. Secondly, the performance of the high chroma backgrounds is substantially worse than the neutral ones (N, D65), which is consistent with Smet's results (Smet, 2017a;Smet, 2017b) that the CAT02 D overestimates the effective degree of adaptation more for chromatic backgrounds. 


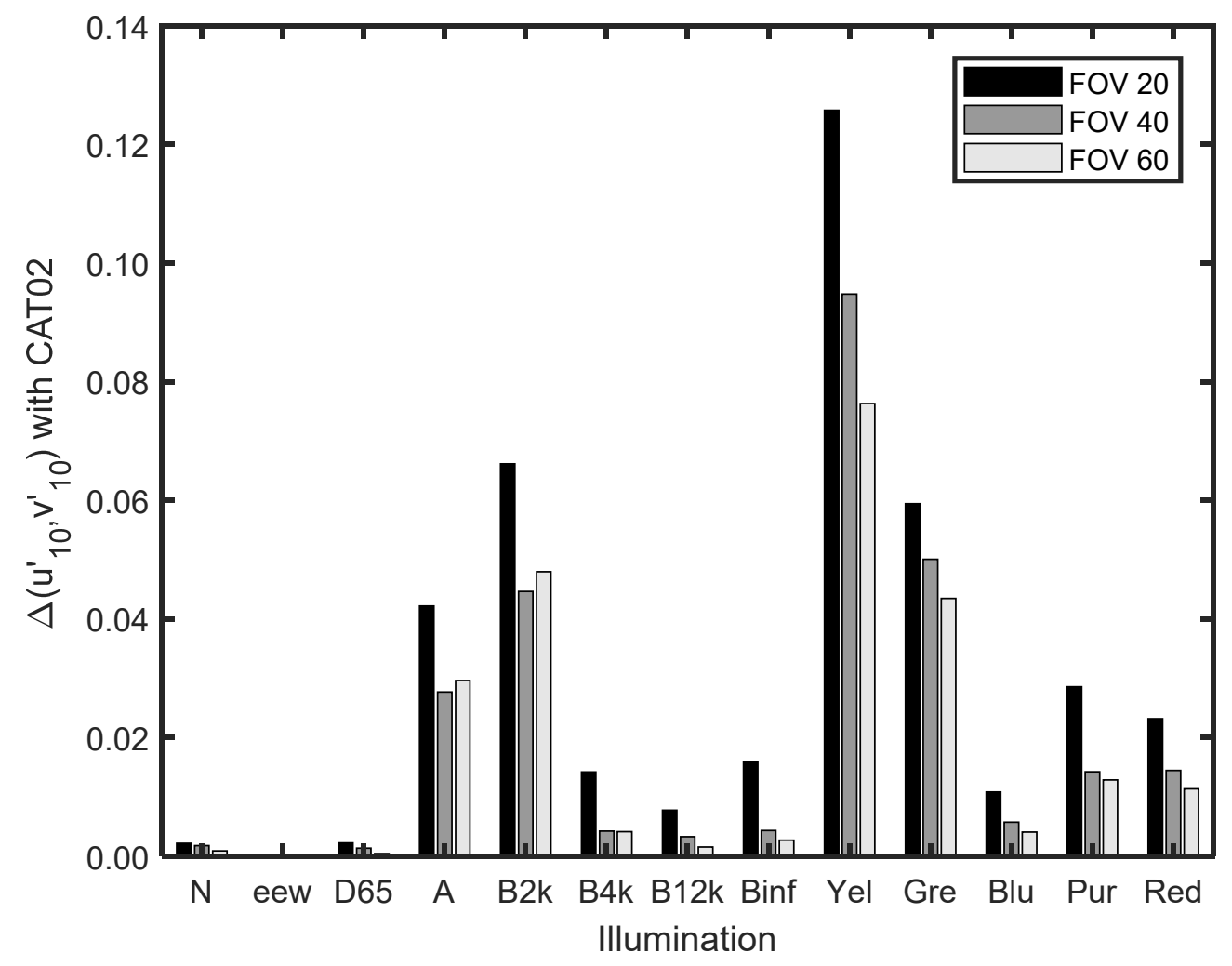

Figure 3 - The prediction error in terms of $\Delta\left(u^{\prime}{ }_{10}, v^{\prime}{ }_{10}\right)$ of CAT02 (with CAT02 sensor and CAT02 D).

\subsection{Optimized $D$ factor}

To minimize the prediction error (colour difference), the $D$ value in CAT02 model was optimized for each background field size and chromaticity. The optimized $D$ is regarded as the effective degree of adaptation under the assumption of a von Kries type chromatic adaptation with CAT02 sensors. Figure $4(a)$ and (b) shows the optimized $D$ and minimized $\Delta\left(u^{\prime} 10, v^{\prime} 10\right)$ for 13 background colours under the three field sizes. Firstly, for almost all the background chromaticities, the optimized $D$ value increases for larger field sizes even though the luminance of the $60^{\circ}$ adapting field is eight times lower than for the $20^{\circ}$ field. Furthermore, the difference between $20^{\circ}$ and $40^{\circ}$ is more substantial than between $40^{\circ}$ and $60^{\circ}$, which may result from a larger proportional difference in field area of the former $(4,00)$ compared to the latter $(2,25)$, or from a diminishing effect of field size at larger sizes (e.g. more peripheral vision contributing less to an observer's adaptation state). Secondly, from Fig. 4(a), it is also clear that the CAT02 $D$ formula typically overestimates the effective degree of adaptation found in this study, especially for the $20^{\circ}$ field size and highly chromatic backgrounds, such as A, B2k, Yellow, Green and Red.

Thirdly, as shown in Figure 4(b), chromaticities A and Gre(en), but especially B2K, have considerably higher prediction error than others, while for neutral (N, D65, B4K) and some bluish (B12K, Blue) chromaticities, the CAT02 model with optimized $D$ performs very well with small $\Delta\left(u^{\prime} 10, v^{\prime} 10\right)(<0,0025)$. The $\Delta\left(u^{\prime}{ }_{10}, v^{\prime}{ }_{10}\right)$ averaged over the 13 background chromaticities for the $20^{\circ}, 40^{\circ}$ and $60^{\circ}$ field sizes are $0,0040,0,0043$ and 0,0037 , respectively. Comparing Figure 4(b) and Figure (3), optimizing the $D$ substantially improves the performance of the corresponding colour prediction of CAT02. From the above, it is clear that both luminance level, chromaticity and adapting field size should be taken into account to predict the effective degree of adaptation more accurately. Finally, even when considering an optimized $D$, for some background chromaticities, such as B2K, the prediction errors with a von Kries type model with CAT02 sensors remains large. This suggests that other sensors could be required or that other additional adaptation (e.g. second site opponent adaptation, contrast adaptation) and vision 
(e.g. simultaneous contrast) processes should be included in a more comprehensive CAT to achieve better general performance.

(a)

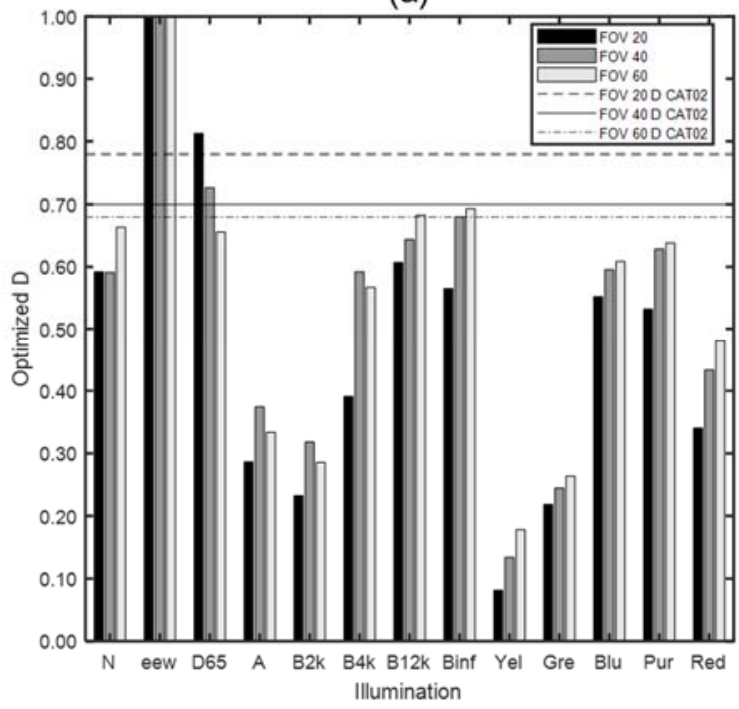

(b)

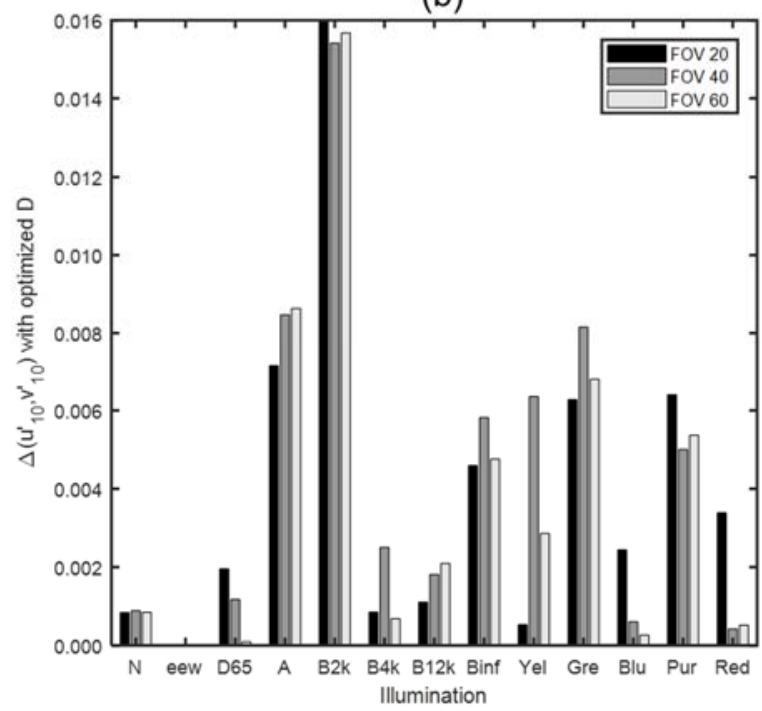

Figure 4 - (a) Optimized $D$ (three bars) resulting in minimized predication error (CAT02 sensor) and CAT02 $D$ (three lines) calculated by Equation (1) for three field sizes

(b) The performance of the CAT02 with optimized $D$ in terms of $\Delta\left(u^{\prime}{ }_{10}, v^{\prime}{ }_{10}\right)$

\section{Conclusion}

An achromatic matching experiment was conducted for 13 background chromaticties under 3 adapting field sizes $20^{\circ}, 40^{\circ}$ and $60^{\circ}$, with equal corneal illuminance level at $7 \mathrm{I} x$. The luminance level of the three sizes correspond to $180 \mathrm{~cd} / \mathrm{m}^{2}, 35 \mathrm{~cd} / \mathrm{m}^{2}$ and $20 \mathrm{~cd} / \mathrm{m}^{2}$.

Firstly, it has been demonstrated that the CAT02 model performs fairly poor for all the background conditions (different chromaticity and field size) because of an overestimation of the effective degree of adaptation. Furthermore, the performance of the CAT02 model is considerably better for the larger background field sizes.

Secondly, at fixed corneal illuminance, a higher degree of adaptation (optimized $D$ ) is achieved with a larger background adaptation field, despite its much lower luminance. However, the differences in optimized $D$ values between $20^{\circ}$ and $40^{\circ}$ are more substantial than those between $40^{\circ}$ and $60^{\circ}$. In addition, it is not only the luminance and field of view, but also the chromaticity of the illuminant that determines the degree of adaptation.

Thirdly, although the optimized $D$ substantially outperforms (smaller prediction error) the CAT02 $D$, prediction errors are still large for more chromatic background chromaticities, such as $\mathrm{A}$ and B2K.

The results obtained in this study indicate the limitation of a von Kries type CAT model with CAT02 sensors and can be adopted to develop a more accurate degree of adaptation formula and a more comprehensive CAT model in the future.

\section{References}

Billmeyer, F.W., and Alessi, P.J. 1981. Assessment of Color $\square$ Measuring Instruments. Color Res. Appl. 6, 195-202.

Brill, M.H., and Mahy, M. 2013. Visualization of mathematical inconsistencies in CIECAM02. Color Res. Appl. 38, 188-195. 
Commission Internationale de l'Eclairage.2004. CIE 160:2004 A Review of Chromatic Adaptation Transforms.

Fairchild, M.D., and Reniff, L. 1995. Time-course of chromatic adaptation for color-appearance judgments. J. Opt. Soc. Am. a-Optics Image Sci. Vis. 12, 824-833.

von Kries, J. 1902. Chromatic adaptation. Festschrift Der Albrecht-Ludwigs-Universität 145158.

Li, C., Li, Z., Wang, Z., Xu, Y., Luo, M.R., Cui, G., Melgosa, M., Brill, M.H., and Pointer, M. 2017. Comprehensive color solutions: CAM16, CAT16, and CAM16-UCS. Color Res. Appl. 42, 703-718.

Li, C., Xu, Y., Wang, Z., Luo, M.R., Cui, G., Melgosa, M., Brill, M.H., and Pointer, M. 2018. Comparing two-step and one-step chromatic adaptation transforms using the CAT16 model. Color Res. Appl. 43, 633-642.

Ma, S., Hanselaer, P., Teunissen, C., and Smet, K.A.G. 2018. THE INFLUENCE OF ADAPTING FIELD SIZE ON DEGREE OF CHROMATIC ADAPTATION. In PROCEEDINGS of CIE 2018 "Topical Conference on Smart Lighting", (Taipei), pp. 161-168.

Moroney, N., Fairchild, M.D., Hunt, R.W.G., Li, C., Luo, M.R., and Newman, T. 2002. The CIECAM02 Color Appearance Model. IS\&T/SID Tenth Color Imaging Conf. 23-27.

Rinner, O., and Gegenfurtner, K.R. 2000. Time course of chromatic adaptation for color appearance and discrimination. Vision Res. 40, 1813-1826.

Smet, K.A.G., Deconinck, G., and Hanselaer, P. 2015. Chromaticity of unique white in illumination mode. Opt. Express 23, 12488.

Smet, K.A.G., Zhai, Q., Luo, M.R., and Hanselaer, P. 2017a. Study of chromatic adaptation using memory color matches, Part I: neutral illuminants. Opt. Express. 25(7), 7732-7748

Smet, K.A.G., Zhai, Q., Luo, M.R., and Hanselaer, P. 2017b. Study of chromatic adaptation using memory color matches, Part II: colored illuminants. Opt. Express. 25(7), 8350-8365. 